\title{
Study of complex systems III-macro-scopic approach on the thermal expansion of deep-fried tofu
}

\author{
Jiro KANAMORI ${ }^{1}$ \\ Fuji Oil Holdings Inc. R\&D Division for Future Creation \\ kanamori.jiro@gmail.com
}

\begin{abstract}
Food processing is a complex system involving a multitude of factors. A new methodology is required to achieve extensive outcomes in such complex systems. In this study, I investigated one such outcome of the expanding properties of deep-fried tofu. Deep-fried tofu samples were made from soy protein isolates (SPI) by mixing, molding, and deep-frying. Expansion of the deep-fried tofu made from different SPIs was correlated to the gel strength of the dough. The sufficient mixing time of each SPI was evaluated, and insufficient mixing resulted in less expansion. The peak height obtained from the electric current curve of the mixer was correlated to the expansion of deep-fried tofu when the mixing was sufficient. A logic tree method could effectively represent the expanding mechanism. This method can be widely applied for analyzing other complex systems.
\end{abstract}

\section{INTRODUCTION}

A new method for understanding and controlling complex systems with many factors is anticipated. Foods are a type of complex system involving a multitude of factors and their combinations. It is crucial to recognize all of these contributing factors in the food industry, because a defective product could be produced because of the lack of even one critical factor. Therefore, a macroscopic approach, in addition to a microscopic approach, is needed for understanding such complex systems. In a previous study, a meso-factor was found to effectively explain the bread-making property (Kanamori, in submission a). In previous work, a logic tree, which is frequently used in executive consulting (Minto, 2009), was found to be applicable for analysis of emulsion foods (Kanamori, in submission b). Although these methods have proven to be useful macroscopic tools, further systematic models will be required for an extensive achievement.

Expansion is a major process of aerated foods. The porous structure resulting from gas

\footnotetext{
1 Current Affiliation: Macro Science Laboratory \#401 SpringTech Tsukuba Building, 2-1-15 Umezono, Tkuba-shi, Ibaraki-ken, JAPAN
} 
contributes to food texture and taste. Expanding mechanisms differ among each type of food. Therefore, this is a complex system with many factors, which remains an open research problem (Campbell and Mougeot 1999).

Deep-fried tofu, or "aburaage", is one of the traditional foods of Japan (Shurtlef and Aoyagi, 2013; Nguyen, 2012). The characteristics of an expanded structure and texture are preferable for deep-fried tofu products, which are widely applied in the production of "wasyoku" (Japanese foods). Tofu is produced by water extraction of swelled soybean followed by heating with divalent metal salts such as magnesium and calcium. The manufacturing of deep-fried tofu consists of an expanding step at $100-120^{\circ} \mathrm{C}$ followed by solidifying the surface at $160-180^{\circ} \mathrm{C}$. The expansion of deep-fried tofu is considered to occur from water vapor. Previous research has suggested that the existence of nucleation of air is necessary for the homogeneous occurrence of vapor (Hashizume et al., 1984a). It was also demonstrated that protein structure, especially disulfide bonds, is an important feature contributing to the expansion rate (Hashizume et al., 1984b).

Deep-fried tofu made from soy protein isolate (SPI) is also commercially produced (Taniguchi, 1987). SPI is a powder that is extracted and isolated from defatted soy meal. Because the deep-fried tofu made from SPI has a simple composition, it serves as a useful model for investigating food expansion.

In this study, the expanding process of deep-fried tofu was investigated in terms of rheology. Several important meso-factors were revealed and sorted into a logic tree. Useful methods for analyzing a complex system are also discussed.

\section{MATERIALS \& METHODS}

Materials

Commercial-grade SPIs (Fujipro E) were obtained from Fuji Oil Co. Ltd. (Osaka, Japan). Other chemicals used were of reagent grade.

\section{Preparation of deep-fried tofu}

Deep-fried tofu was prepared by mixing, molding, and deep-frying, and the size was measured to calculate the index of expansion. The dough consisted of $88.4 \mathrm{~g}$ SPI, $26.5 \mathrm{~g}$ rapeseed oil, $1.3 \mathrm{~g} \mathrm{CaSO}_{4} 2 \mathrm{H}_{2} \mathrm{O}, 0.88 \mathrm{~g} \mathrm{NaCl}$, and $283 \mathrm{~g}$ water, which was mixed by a cutting-mixer (MK-K72, Matsushita Electric Co. Ltd., Japan) for $4 \mathrm{~min}$ at $20^{\circ} \mathrm{C}$. The rotation speed was $3200 \mathrm{rpm}$ during an idle run. Molding was performed by casing followed by cutting. The dough was poured into a plastic casing tube of $22 \mathrm{~mm}$ in diameter, the end was clipped, and it was left to stand for $5 \mathrm{~min}$ to solidify. The casing tube was then removed and the elastic dough was cut into disks of 6-mm thickness. The molded dough was fried in rapeseed oil in the order 
of $70^{\circ} \mathrm{C}$ for $4 \mathrm{~min}, 110^{\circ} \mathrm{C}$ for $1.5 \mathrm{~min}$, and $175^{\circ} \mathrm{C}$ for $3.5 \mathrm{~min}$. After $30 \mathrm{~min}$ of cooling in the air, the diameter of deep-fried tofu was measured in the perpendicular direction and then averaged. The expanding size was calculated as the area of deep-fried tofu assuming a circle. Six samples were analyzed for each dough type, and linear regression was performed using the Microsoft Excel function.

\section{Gel strength}

The dough mixed in the same manner as that described for preparation of the deep-fried tofu was deformed by vacuuming, poured in a plastic casing tube, and heated at $80^{\circ} \mathrm{C}$ for $30 \mathrm{~min}$ in a water bath. After cooling overnight at $4{ }^{\circ} \mathrm{C}$, the gel was removed from the casing tube, cut into cylinders of $20-\mathrm{mm}$ height, and the gel strength was measured at $20^{\circ} \mathrm{C}$ using a creep meter (RE33005, Yamaden Co. Ltd., Tokyo, Japan) with a 5-mm ball plunger at $1 \mathrm{~mm} / \mathrm{s}$. The gel strength was measured as the breaking force. Five samples were analyzed for each SPI, and linear regression was performed using the Microsoft Excel function.

\section{Mixing property}

Electric current curves of dough mixing were obtained during the mixing process of each deep-fried tofu sample using an ammeter (Clamp on Leak Hitester 3283; Hioki E.E. Co., Nagano, Japan) and a chart recorder (PRR-5021; DKK-TOA Co., Tokyo, Japan), as described previously (Kanamori, 2016). Peak height and peak time were obtained as mixing parameters. Duplicate measurements were performed.

\section{RESULTS AND DISCUSSION}

Relationship between gel strength of the dough and deep-fried tofu expansion

The relationship between gel strength of the dough and deep-fried tofu expansion was investigated. The expansion of deep-fried tofu samples from different SPIs was plotted against the gel strength of each dough (Fig. 1). The linear regression with a correlation coefficient $\left(\mathrm{R}^{2}=\right.$ 0.80 ) was observed between the gel strength values and the sizes of deep-fried tofu samples.

Hashizume et. al. (1984a, b) indicated that the expansion of deep-fried tofu made from whole soybean was driven by steam vapor. This seems to be the same expanding mechanism of deep-fried tofu made from the SPIs in this study, because visual observation clearly showed that the expansion began at the time of steam generation. It is well known among food processors that larger deep-fried tofu is generally obtained with stiffer dough; however, no direct evidence of this relationship has been reported. Hashizume et. al. (1984 b) investigated the free sulfhydryl group $(\mathrm{SH})$ content of the protein and gel strength, and revealed that the optimum $\mathrm{SH} /$ disulfide 
bond (SS) ratio of the residue was an important factor for gel strength. They also revealed that the $\mathrm{SH} / \mathrm{SS}$ ratio was less important for deep-fried tofu expansion and there was no direct relationship between gel strength and deep-fried tofu expansion.

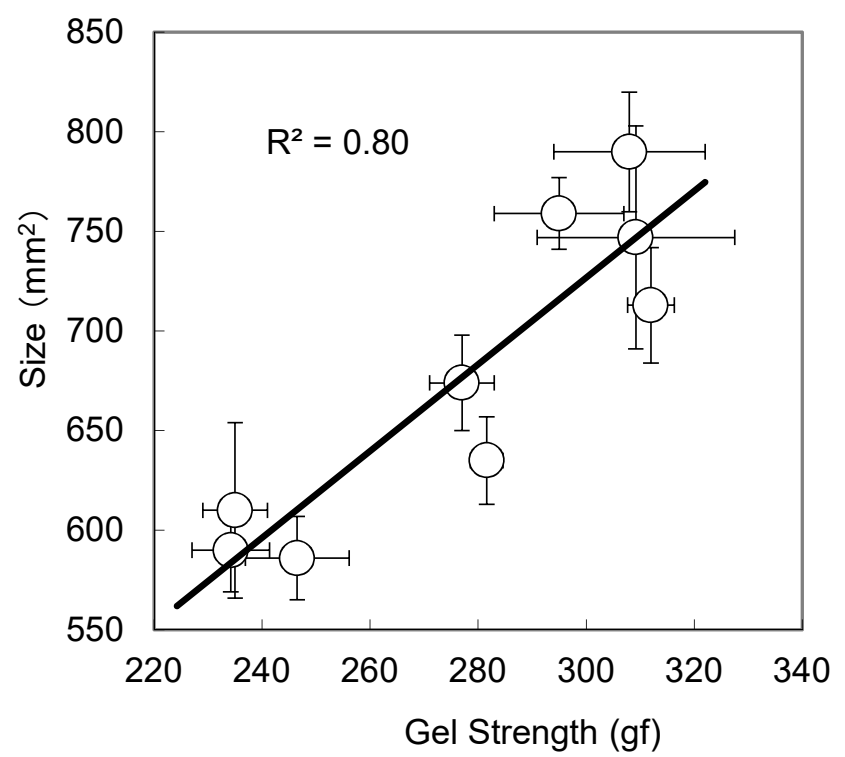

Fig. 1 Relationship between gel strength of the dough and expansion of deep-fried tofu

Based on the results of the present study, an expansion mechanism was proposed, as illustrated in Fig. 2. Deep-fried tofu is expanded by steam vapor, which generates air bubbles in the dough (Fig. 2a) (Hashizume et. al., 1984a). The dough gel by heating at steam generation temperature $\left(100^{\circ} \mathrm{C}\right)$ (Fig. $\left.2 \mathrm{~b}\right)$. The vapor volume is much larger than the dough volume; therefore, the dough extends until the breaking point and stops deforming by losing steam pressure (Fig. 2c). The expansion phenomenon of deep-fried tofu can be interpreted as a type of stress-deformation, similar to a gel breaking test. Therefore, it is reasonable that the gel strength corresponded to the extent of deep-fried tofu expansion (Fig. 1). The dough is fixed by surface drying during deep-frying, which no longer affects expansion (Fig. 2d).

Rheological properties are frequently related to the expansion of a solid or viscous food such as bread dough. Bread expansion consists of several factors: generation of carbon dioxide during fermentation, thermal expansion of air and carbon dioxide, and generation of steam vapor during baking (Campbell and Mougeot, 1999). The latter stage of oven-rise has a significant effect on the quality of bread (Rosell et al., 2001). Each expanding process differs in temperature and deformation velocity. Therefore, a rheological experiment conducted at the 
same condition of the expanding processes would help to reveal the more precise mechanism.

(a)

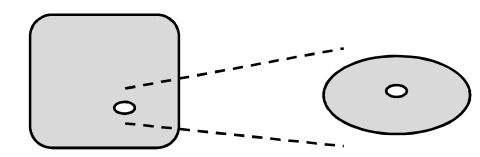

(b)

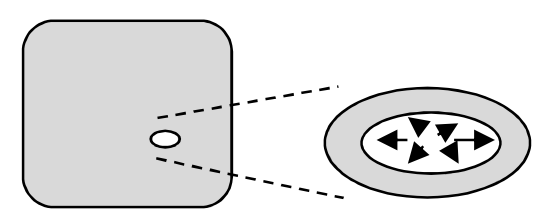

(c)

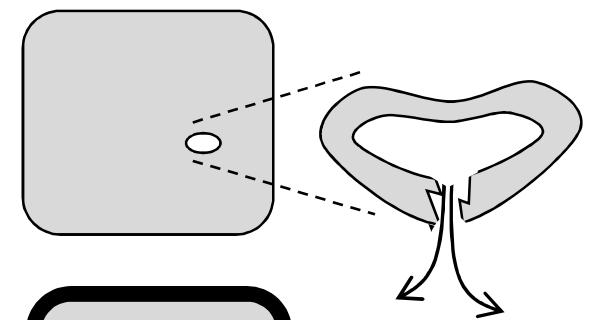

(d)

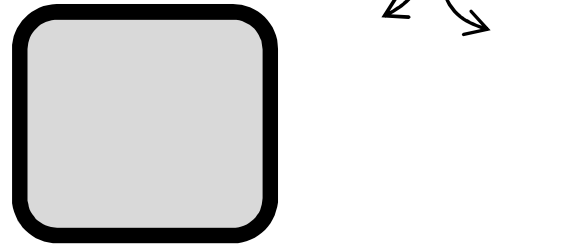

\section{Fig. 2 Illustration of the expanding mechanism of deep-fried tofu}

The left column represents the whole dough and the right column is an enlarged view of a portion of the dough. (a) The dough does not expand under the boiling point of water. (b) Over the boiling point, water vapor outbreaks at air bubbles and extends the dough. (c) When the extension reaches its limit, the dough breaks and water vapor escapes out of the dough. The dough expansion stops because the driving force disappears. (d) The dough surface is deep-fried at $160^{\circ} \mathrm{C}$ and the expanded structure is fixed.

\section{Relationship between mixing properties and expansion of deep-fried tofu}

The relationship between mixing properties and expansion of the deep-fried tofu was examined. Two different SPIs with similar peak heights and different peak times were used (SPI-A: peak time $33 \mathrm{~s}$ and peak height 2.0 A; SPI-B: peak time $78 \mathrm{~s}$ and peak height $1.8 \mathrm{~A}$ ). When the deep-fried tofu samples were made from the dough at various mixing periods, the 
expansion increased as the mixing time increased (Fig. 3). Larger expansion was obtained when the dough was mixed for longer periods. Expansion reached a steady state at over $120 \mathrm{~s}$ for SPI-A. SPI-A required a shorter mixing period than SPI-B, which indicated that the developing speed of SPI-A was higher than that of SPI-B.

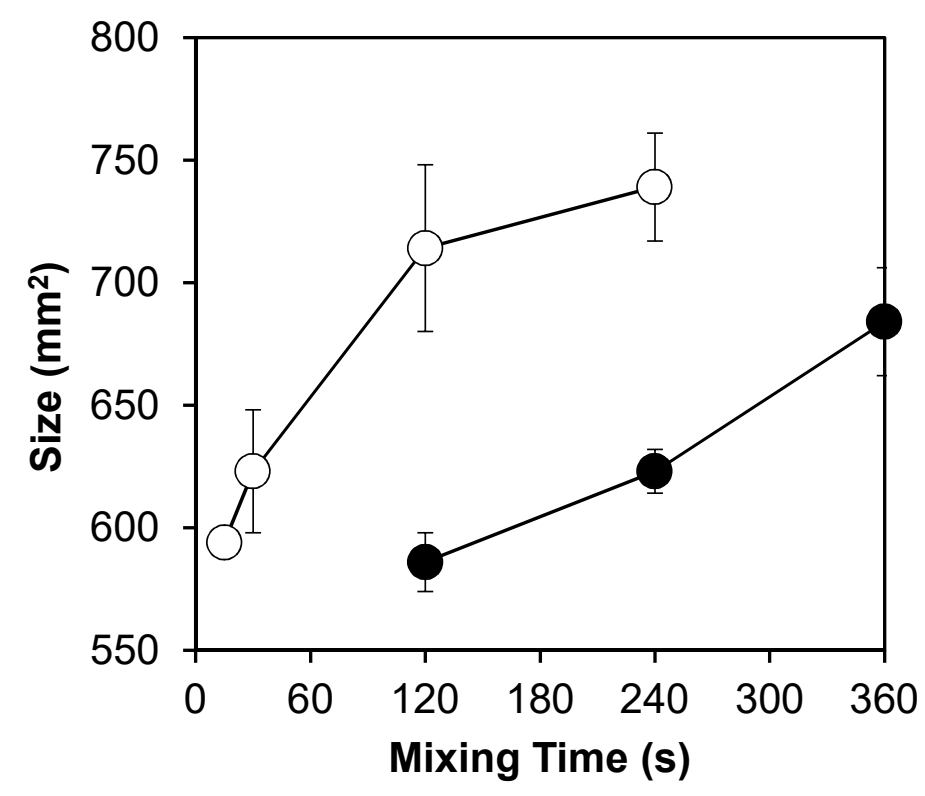

Fig. 3 Effect of mixing time on expansion of deep-fried tofu

Open circles: sample A with a relatively low peak time. Closed circles: sample B with a relatively high peak time.

A weak correlation $\left(\mathrm{R}^{2}=0.63\right)$ was observed between the peak height and expansion of deep-fried tofu (Fig. 4a); however, when the dough with over $45 \mathrm{~s}$ peak time were excluded, a very strong correlation $\left(\mathrm{R}^{2}=0.98\right)$ was observed between the peak height and expansion of deep-fried tofu (Fig. 4b). The correlation between gel strength and peak height was also found in the previous study (Kanamori, 2016). Therefore, dough with a longer peak time would mix poorly at an appropriate mixing time, and the potential expanding ability may not be exhibited.

The gel strength of the dough was not highly correlated with the peak height (data not shown). Gel strength differs depending on the measurement conditions such as plunger type, velocity, protein concentration, and heating temperature. Expansion of deep-fried tofu is a deformation phenomenon at high temperatures of $100^{\circ} \mathrm{C}$; therefore, the optimal deformation conditions of temperature and deforming velocity significantly differ from those used for the measurement of gel strength. Although the mixing-recording experiment was also performed at different measurement conditions, it was remarkable that the correlation between the peak 
height and expansion was high. Extensive studies will be required to elucidate the relationship among the mixing, gelation, and expanding properties of deep-fried tofu.

(a)

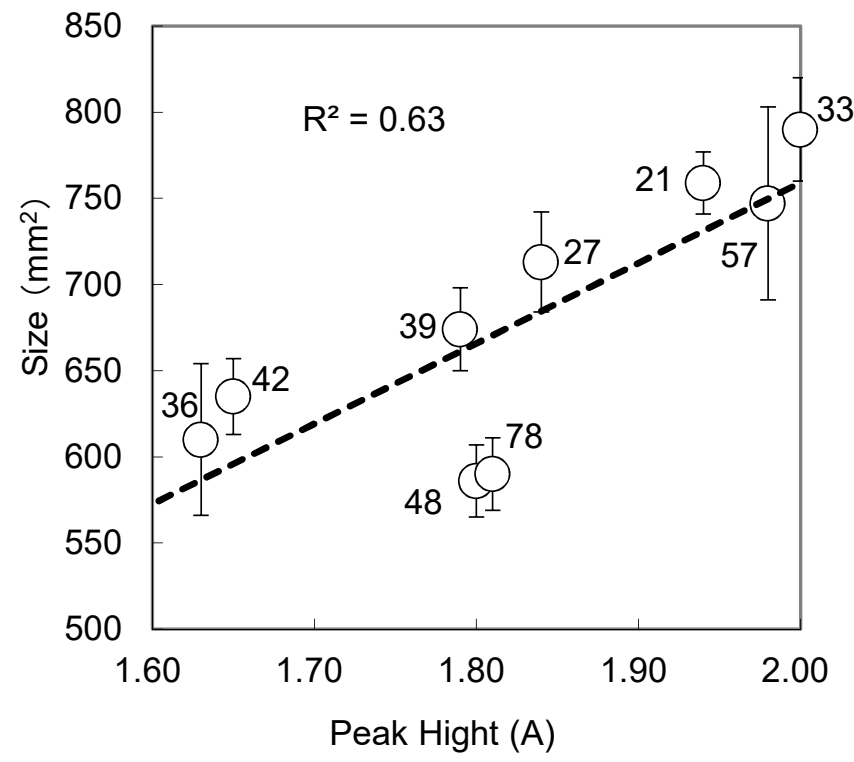

(b)

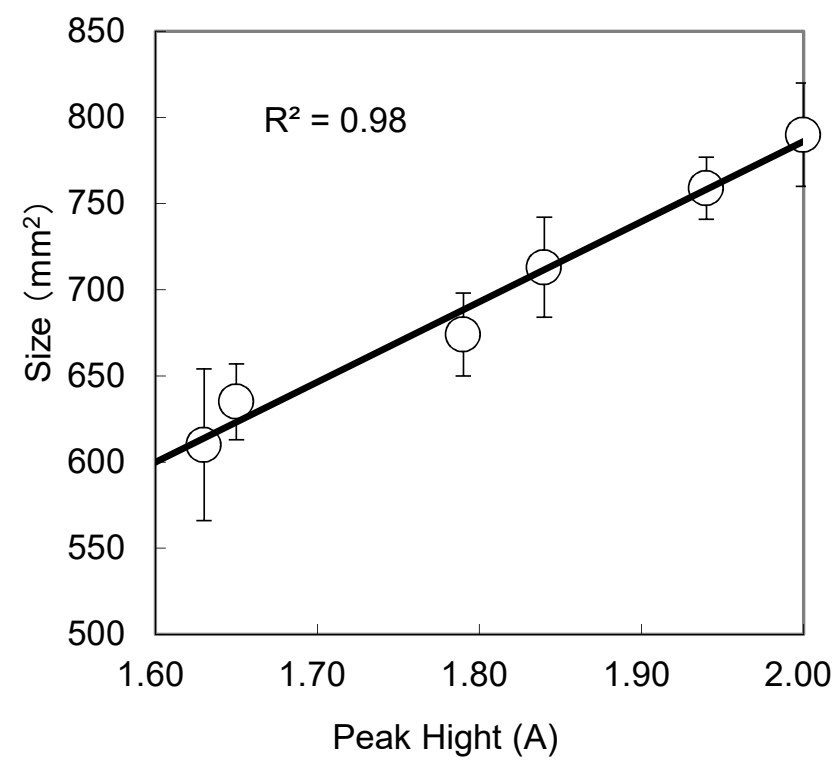

Fig. 4. Relationship between peak height and expansion

(a) All samples, (b) Samples with a relatively low peak time

Factors involved in the expansion of deep-fried tofu

In this study, a mechanical interpretation is proposed about the expansion of deep-fried 
tofu made from SPI and its relationship with mixing characteristics. The factors involved in the expansion of deep-fried tofu were organized in a logic tree (Fig. 5)

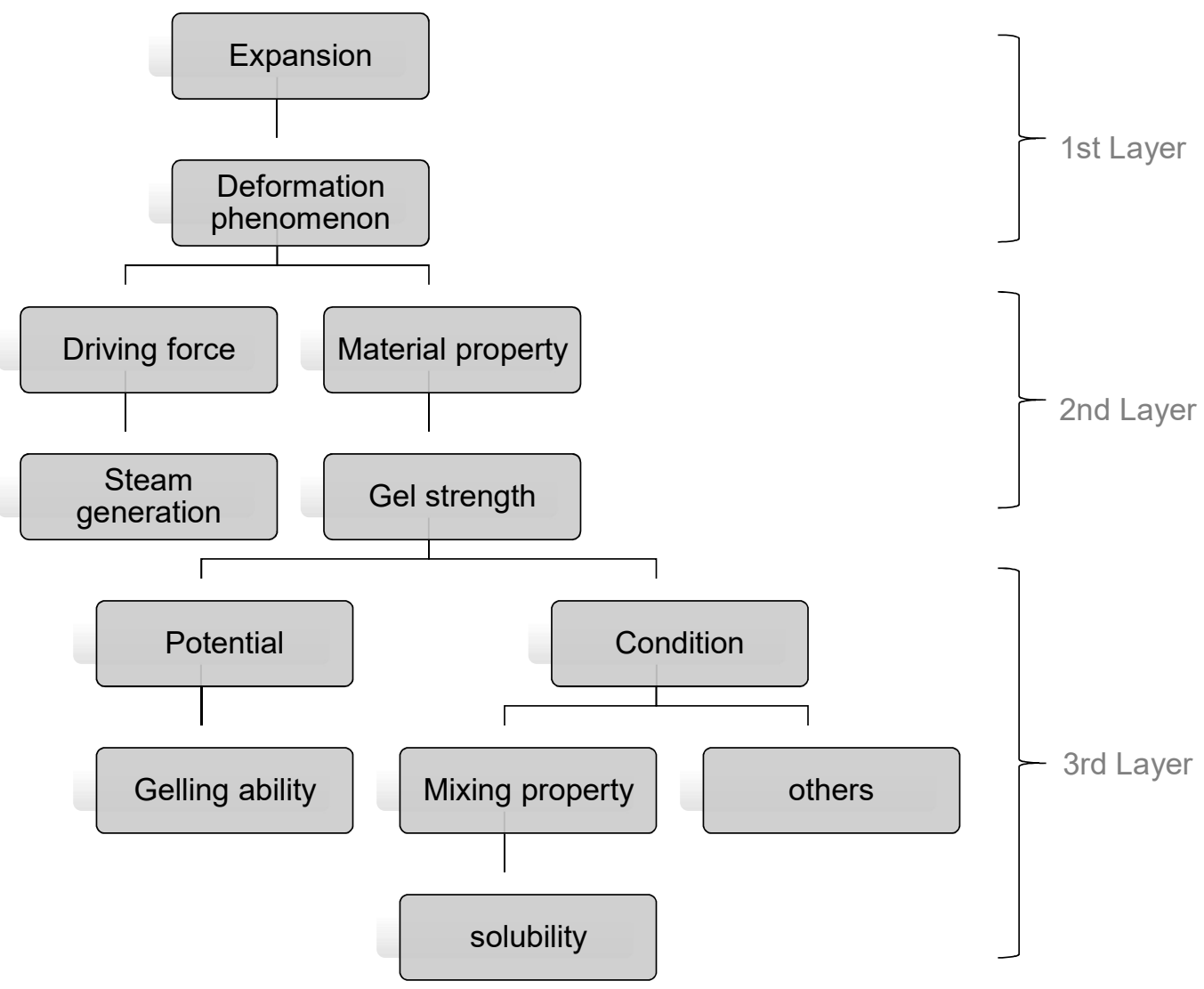

Fig. 5. Logic tree of the factors affecting the expansion of deep-fried tofu

In the first layer, the expansion of deep-fried tofu was interpreted as a mechanical deformation phenomenon. This interpretation made it possible to divide the second layer into two factors: driving force and material property. The former was found to result from the occurrence of water vapor in the previous study (Hashizume et. al., 1984a), and the latter was interpreted as the gel strength based on the experimental results in the present study (Fig. 1, Fig. 2). These "interpretations" were extremely important, because they suggest that the expansion-a natural phenomenon-could be translated to rheological measurement, with a substantial supporting scientific basis. To estimate the raw material, the gel strength was divided into the potential and the condition at the third layer. The former was interpreted as the gel 
forming ability, and one of the latter factors was mixing properties which was known to be associated with solubility (Kanamori, 2016). The expanding properties of deep-fried tofu are derived from the mechanical properties of SPI, and two basic mechanical properties-gelation property and solubility-were summarized from the results of this study. These properties were meso-factors, which include many other factors such as components, molecular structures, and processing conditions. Only a few studies have investigated the expansion of deep-fried tofu, whereas many studies have addressed the gelation and solubility of soy protein (Kinsella 1979; Morr 1990). The expanding property was summarized into the two basic properties of SPI in this study; therefore, we can now use results of previous studies on soy protein to better understand the process of deep-fried tofu expansion. Furthermore, most of the factors were collected in the second and third layers; they were mutually exclusive and collectively exhaustive (MECE) relations between the driving force and the material property in the second layer, and between the potential and the condition in the third layer (Minto, 2009). Because it is important to express the whole system, the concept of MECE partitioning is useful in addition to taking a meso-factor approach.

These two parameters of SPI were possibly measured as peak height and peak time. This method will be a great industrial advantage for controlling the quality of deep-fried tofu. Moreover, the method proposed in this study using a logic tree with "interpretations", meso-factors, and MECE partitions is widely applicable for the analysis of other complex systems.

\section{CONCLUSION}

Expansion of deep-fried tofu was investigated. The expansion was correlated with gel strength of the dough and affected by the mixing state of the dough. The logic tree with "interpretations", meso-factors, and MECE partitions simplified these complex factors, and appears to be a useful tool to analyze complex systems.

\section{ETHICAL STATEMENTS}

Conflict of Interest: The author declares that he has no conflict of interest.

Ethical Review: This study did not involve any human or animal testing.

\section{REFERENCES}

Campbell, G. M. and Mougeot, E., 1999. Creation and characterisation of aerated food products. Trends in Food Science \& Technology, 10, 283-296. doi:10.1016/S0924-2244(00)00008-X

Hashizume, K., Saio, K. and Ikeda, T., 1984a. Studies on Abura-age [fried bean curd]-making, 
1: The effects of air content in soybean milk on the expansion of Tofu [bean curd]-gels for Abura-age-making. Nippon Shokuhin Kogyou Gakkai-Shi, 31, 389-394 (in Japanese) doi:10.3136/nskkk1962.31.6_389

Hashizume, K., Saio, K. and Ikeda, T., 1984b. Studies on Abura-age [fried bean curd]-making, 2: The relationship of SH and S-S exchange reaction to the expansion of Tofu [bean curd]-gels for Agura-age-making. Nippon Shokuhin Kogyou Gakkai-Shi,, 31, 395-400 (in Japanese) doi:10.3136/nskkk1962.31.6_395

Kanamori, J. (2016). Mixing-recording method for the practical determination of physical properties of soy protein. Cereal Chemistry, (in press).

doi:dx.doi.org/10.1094/CCHEM-12-15-0258-R

Kanamori, J., in submission a. Study of complex food systems I-filler effect as a meso-factor of bread making property.

Kanamori, J., in submission b. Study of complex food system II-utilization of a logic tree for physical properties of an emulsion food.

Kinsella, J. E., 1979. Functional properties of soy proteins. Journal of the American Oil Chemists' Society, 56, 242-258. doi:10.1007/BF02671468

Minto, B., 2009. The pyramid principle: logic in writing and thinking. Pearson Education, Harlow.

Morr, C. V., 1990. Current status of soy protein functionality in food systems. Journal of the American Oil Chemists' Society, 67, 265-271. doi:10.1007/BF02539674

Nguyen, A., 2012. In “Asian Tofu: Discover the Best, Make Your Own, and Cook It at Home”, Random House LLC, New York, pp. 11.

Rosell, C. M., Rojas, J. A., and De Barber, C. B., 2001. Influence of hydrocolloids on dough rheology and bread quality. Food Hydrocolloids, 15, 75-81.

doi:10.1016/S0268-005X(00)00054-0

Shurtleff, W. and Aoyagi, A., 2013. In "History of Tofu and Tofu Products “, Soyinfo Center, 
Lafayette, pp. 952-960.

Taniguti, H., 1987. Popularization of New Soy Protein Products in Japan. In "Proceeding of the Satellite Sympojium on Soy Protein in Human Nutrition”, Inoue, G., ed., Research Committee Soy Protein Nutrition, Japan, pp. 89-101 